\title{
Differences in Hypertension Medication Prescribing for Black Americans and Their Association with Hypertension Outcomes
}

\author{
Hunter K. Holt, MD, MAS, Ginny Gildengorin, PhD, Leab Karliner, MD, MAS, \\ Valy Fontil, MD, MAS, MPH, Rajiv Pramanik, MD, and Michael B. Potter, MD
}

Background: National guidelines recommend different pharmacologic management of hypertension (HTN) without comorbidities for Black/African Americans (BAA) compared with non-BAA. We sought to 1) identify if these recommendations have influenced prescription patterns in BAA and 2) identify the differences in uncontrolled HTN in BAA on different antihypertensive medications.

Methods: We constructed a linked retrospective observational cohort using 2 years of electronic health records data, comprising of patients aged 18 to 85 with HTN on 1- or 2-drug regimens, including angiotensin-converting enzyme inhibitors (ACE), angiotensin receptor blockers (ARB), thiazide diuretics, or calcium channel blockers (CCB). We examined prescribing differences and HTN control in BAA versus non-BAA.

Results: Among 10,875 patients identified, $20.6 \%$ were identified as BAA. 46.4\% of BAA had uncontrolled HTN $(\geq 140 / 90 \mathrm{mmHg})$ compared with $39.0 \%$ of non-BAA $(P<.001) .61 .8 \%$ of BAA were treated with 1-drug compared with $68.4 \%$ of non-BAA. Of BAA on monotherapy: $41.3 \%$ were on thiazide, $40.1 \%$ on CCB, and $18.6 \%$ on ACE/ARB. Of non-BAA on monotherapy, $27.7 \%$ were on thiazide, $30.1 \%$ were on CCB, and $42.3 \%$ were on ACE/ARB. Of BAA patients on 1 drug, $45.2 \%$ had uncontrolled HTN compared with $38.0 \%$ of non-BAA $(P<.001)$. Of BAA on 2 drugs, $48.2 \%$ had uncontrolled HTN compared with 41.1\% non-BAA $(P<.001)$. For each drug regimen, there was more variation in HTN control within each group than between BAA and non-BAA.

Conclusions: Providers seem to be following race-based guidelines for HTN, yet HTN control for BAA remains worse than non-BAA. An individualized approach to HTN therapy for all patients may be more important than race-based guidelines. ( $\mathrm{J}$ Am Board Fam Med 2022;35:26-34.)

Keywords: Health Equity, Hypertension, Precision Medicine, Prescriptions, Primary Health Care, Retrospective Studies

\section{Background}

Race-based guidelines for medical care have recently become controversial. ${ }^{1-7}$ Skin color is increasingly

This article was externally peer reviewed.

Submitted 6 July 2021; revised 24 August 2021; accepted 25 August 2021.

From the Department of Family and Community Medicine at the University of Illinois at Chicago (HKH); Department of Family and Community Medicine, University of California San Francisco (MBP); Division of General Internal Medicine, Department of Medicine, University of California San Francisco (GG, LK, VF); Multiethnic Health Equity Research Center, University of California San Francisco (LK, VF); UCSF Center for Vulnerable Populations at Zuckerberg San Francisco General Hospital, San Francisco, CA (VF); Contra Costa Health Services, San Francisco, CA (RP).

Funding: This research was supported by the National Center for Advancing Translational Sciences, National Institutes of Health (UL1 TR001872). JKH was supported recognized as a poor proxy for precision medicine, and many have highlighted the potential harms of using race as a factor in clinical decision-making. ${ }^{4,7-10}$ For example, well-intentioned medical organizations, relying on data from clinical trials, have long recommended different standards for interpreting kidney and pulmonary function testing for Black/African

by the National Research Service Award (NRSA) grant (T32HP19025).

Conflict of interest: The authors declare that they have no conflicts of interest.

Availability of data and materials: The study protocol, raw data, and/or programing code supporting the conclusions of this article are available from the corresponding author on reasonable request.

Corresponding author: Michael B. Potter, MD, University of California, San Francisco 505 Parnassus Ave, San Francisco, CA 94143; Phone: 415-476-1000 (E-mail: michael.potter@ucsf.edu). 
Americans (BAA) compared with others, even as many have suggested that relying on such guidelines may lead to delayed diagnosis and treatment for BAA populations. ${ }^{2,6,11-14}$ In this context, it may be valuable to reevaluate widely accepted race-based guidelines for managing hypertension (HTN) in BAA. ${ }^{15}$ The Eighth Joint National Committee in 2014 (JNC8), the American College of Cardiology/ American Heart Association in 2017 (ACA/AHA), and European Society of Cardiology 2018 guidelines all recommend a unique approach to initial therapy for BAA, stating that individuals in this group without comorbidities should receive either a thiazide diuretic or a calcium channel blocker (CCB) as initial therapy, whereas angiotensin-converting enzyme inhibitors (ACE) and/or angiotensin receptor blockers (ARB) should be considered as initial therapy only for BAA with certain comorbidities while non-BAA individuals can be prescribed any of those 3 classes of medicines regardless of comorbidities. ${ }^{16-18}$ These guidelines echoed sentiments in the International Society on Hypertension in Blacks (ISHB) consensus statement in 2010 that recommended that first-line treatment consist of a thiazide diuretic or CCB over an ACE/ ARB in BAA populations, but not other populations. ${ }^{19}$

Race-based HTN treatment guidelines have been based on evidence from clinical trials and intended to rectify disparities in HTN control in BAA populations. $^{20,21}$ However, the interpretation of this evidence has come under scrutiny. ${ }^{15}$ JNC8 and ACA/ AHA recommendations are based on results from the Anti-HTN and Lipid-Lowering treatment to Prevent Heart Attack Trial (ALLHAT) and the African American Study of Kidney Disease and Hypertension study (AASK). ALLHAT found in the primary outcome of coronary heart disease there was no difference for BAA when using thiazide, CCB, or ACE. While based on a prespecified secondary outcome of strokes, there was a significant benefit from using $\mathrm{CCB} /$ thiazide medications compared with ACE for BAA. However, this finding has been challenged, as the treatment regimen for patients on ACE included a $\beta$ blocker, which is no longer considered an appropriate regimen for any patient with HTN and no other comorbidities. ${ }^{15,22}$ The AASK Trial evaluated BAA populations with chronic kidney disease and the effects of medications in those populations. While ACE was not as effective at lowering blood pressure (BP) in BAA, it was found that they did prevent the progression of kidney disease. ${ }^{23}$ It has been pointed out that avoiding ACE to treat
BAA with uncomplicated HTN could lead to worse outcomes for those whose progression of kidney disease is not immediately recognized in primary care. $^{15,24}$

Given the current concerns about health equity in the United States, we must examine whether and how race-based guidelines for HTN treatment have influenced the practice patterns of primary care clinicians and whether there is evidence of a beneficial impact from following such guidelines on clinical outcomes for BAA with HTN. In this study, our objective was to evaluate electronic health record (EHR) data to identify if these race-based guidelines have influenced health care provider prescribing practices in BAA groups and evaluate the HTN control for BAA and non-BAA patients with uncomplicated HTN who were prescribed 1 or 2 drugs. We additionally examined predictors of uncontrolled HTN among BAA patients.

\section{Methods}

\section{Study Design}

This study is a retrospective, observational cohort analysis using 2 years of linked EHR data from 3 health systems affiliated with the San Francisco Bay Collaborative Research Network (sfbaycrn.org). The 3 health systems were comprised of 1 academic and 2 county-run systems with a total of 31 primary care clinics. The study included all patients aged 18 to 85 years at the beginning of the study period with a hypertension diagnosis and at least 1 primary care provider care visit between October 15, 2013, and October 14, 2015, for 2 of the 3 health systems, and between May 1, 2014, and April 30, 2016, for the third health system. These 2-year periods were different due to local constraints and Health System 1 to pull data retrospectively to match the dates from the other 2 health systems. All the data were collected using a 1-time extraction of EHR data. HTN diagnosis in each was defined using the Ninth International Classification of Disease visit diagnosis codes 401 and 405 during the study period or a problem list diagnosis indicating "hypertension." Initial exclusion criteria during data extraction were pregnancy-related HTN and endstage renal disease.

\section{Study Population}

For this subset analysis, inclusion criteria encompassed: 1) patients on 1-drug regimens that 
included either ACE/ARB, thiazide, or CCB; 2) patients on 2-drug regimens on ACE/ARB and/or thiazide and/or CCB. Exclusion criteria included: individuals with diabetes, chronic kidney disease (CKD), not currently on any HTN medications, on 3 or more HTN medications, or 1- or 2-drug regimens including drugs other than ACE, ARB, thiazide, or CCB. Patients with diabetes and CKD were excluded as we did not have data on microalbuminuria, a case in which ACE/ARB are indicated as the first line in BAA populations per national guidelines. Patients on 3 or more medications were excluded as this meets the diagnosis of resistant HTN and is outside the scope of this study. Finally, patients on other medications other than ACE/ $\mathrm{ARB}$, thiazide, or CCB were excluded as they are not recommended for initial HTN therapy in any guideline recommendations.

\section{Measurements}

The primary outcome was prescription patterns in BAA and non-BAA groups. Our secondary outcome of interest was uncontrolled HTN at a patient's most recent primary care visit, which we defined as a BP reading of stage $2 \mathrm{HTN}$ or greater $(\geq 140 / 90$ $\mathrm{mmHg}$ ). Controlled HTN was considered Stage 1 HTN or below $(<140 / 90 \mathrm{mmHg})$. We chose this cutoff because there are multiple recommendations for optimal BP for adults, elderly adults, and BAA in particular. JNC8 guidelines recommend that for adults 18 to 59 years of age, $<140 / 90 \mathrm{mmHg}$ is the ideal goal, while adults 60 years or older without comorbidities should have a treatment goal of $<150 / 90$ mmHg. ${ }^{16}$ ACA/AHA guidelines recommend a treatment goal for all adults to be $<130 / 80$ mmHg regardless of age. ${ }^{17}$ The European Society of Cardiology recommends for adults aged 18 to 59 years, treatment goals should be $<130 / 90 \mathrm{mmHg}$, and for adults 60 years and older, the goal should be $<140 / 90$ mmHg. ${ }^{18}$ Finally, the ISHB consensus statement recommends a goal $\mathrm{BP}$ of $<135 / 85$ $\mathrm{mmHg}$ in BAA without comorbidities in all ages. We chose our cutoff for all ages because it represented a compromise between the different guidelines, balancing the more stringent ACA/AHA guidelines and the relatively liberal JNC8 guidelines for different age groups. Furthermore, clinician leaders representing these practices settings in our practice-based research network agreed that this cutoff represents a level of control that would be expected to trigger additional intensified medical therapy.

We also collected race and ethnicity (as identified in the EHR), age, sex, body mass index (BMI, calculated from height and weight data), smoking status, preferred language, insurance status, and number of primary care visits during the 2-year study period. Age, BMI, smoking status, and insurance status were collected for the first visit during the study period. For the purpose of this study, a list of all the medications listed as currently prescribed for HTN was pulled in the 1-time extraction of EHR data at the last visit available. Medications (including combination drugs) were broken down into respective medicines and classes.

\section{Analysis}

We reported population characteristics stratified by BAA population and non-BAA population. Initial analyses evaluated proportion of cohorts with controlled HTN or uncontrolled HTN on 1- and 2drug regimens using the proportional z-test. In addition, we examined median systolic and diastolic BP (SBP and DBP) for all participants on each possible treatment regimen, stratified by $\mathrm{BAA}$ and non-BAA. We used Pearson's $\chi^{2}$ test for bivariate analysis of categorical data and Student's $t$-test for continuous data. Multivariate logistic regression models were used to evaluate the association of uncontrolled HTN for both 1- and 2-drug regimens in the BAA subgroup with the following covariates: age as a continuous variable, insurance status (categorical variable: insured, uninsured, and unknown), number of primary care provider (PCP) visits over the 2-year study period (categorical variable: 1 to 2, 3 to 4 , or $\geq 5$ visits), and health system where primary care was delivered.

All analyses were performed with SAS 9.4 and STATA 16 data analytics software. The value of $P=.05$ was used as the cutoff level for statistical significance. Further details on our methods to define our research cohorts and extract/clean/link data from the EHR data can be found in an earlier publication. $^{25}$ The Institutional Review Board approved the study at the University of California, San Francisco, with a waiver for informed consent.

To improve transparency, we followed the REporting of studies Conducted using Observational Routinely collected health Data (RECORD) guidelines when presenting our study. ${ }^{26}$ 


\section{Results}

Sociodemographic and Baseline Characteristics

For our subset analysis, 10,875 participants met inclusion criteria. Mean age for this cohort was 56.2 (standard deviation [SD], 12.0) years. The majority $(57.8 \%)$ of the cohort were female, $94.0 \%$ had some form of health insurance (private, Medicare, or Medicaid), and $18.3 \%$ were current smokers. One-fifth $(20.6 \% ; n=2245)$ of the cohort were identified as BAA in the EHR. Of note, a higher proportion of BAA were smokers $(38.7 \%)$ compared with non-BAA $(13.0 \%)(P<.001)$, and a higher proportion of BAA was obese $(\mathrm{BMI} \geq 30)$ $(52.2 \%)$ as compared with non-BAA (34.9\%) $(P<.001)$. (Table 1$)$.

\section{Pharmacological Treatment Characteristics}

Of the total cohort, $67.1 \%$ were on 1-drug regimens and $32.9 \%$ were on 2-drug regimens. Among BAA patients, $61.8 \%$ were on 1-drug and $38.2 \%$ were on 2-drug regimens; whereas $68.4 \%$ of non-BAA patients were on 1-drug regimens and $31.6 \%$ were on 2-drug regimens. Among BAA patients on 1-drug regimens: $41.3 \%$ were on thiazide, $40.1 \%$ on $\mathrm{CCB}$, and $18.6 \%$ on ACE/ARB. Among non-BAA patients on 1-drug regimens: $27.7 \%$ were on thiazide, $30.1 \%$ on $\mathrm{CCB}$ and $42.3 \%$ on ACE/ARB. Among BAA patients on 2-drug regimens: $35.8 \%$ were on thiazide $+\mathrm{CCB}, 44.3 \%$ on thiazide $+\mathrm{ACE} / \mathrm{ARB}$ and $19.8 \%$ were on $\mathrm{CCB}+\mathrm{ACE} / \mathrm{ARB}$. Among non-BAA patients on 2-drug regimens: $19.8 \%$ were on thiazide $+\mathrm{CCB}, 49.4 \%$ on thiazide $+\mathrm{ACE} / \mathrm{ARB}$ and $30.8 \%$ on $\mathrm{CCB}+\mathrm{ACE} / \mathrm{ARB}$. (Table 2)

\section{Median Blood Pressure by Race and by Drug Regimen}

Median SBP and DPB for each treatment regimen for BAA and non-BAA populations did not exceed $140 \mathrm{mmHg}$ or $90 \mathrm{mmHg}$. (Figures 1a and 1b) There were minimal between-group (BAA vs non-BAA) differences in median blood pressure for any regimen. There was some within-group variation. For BAA patients on 1-drug regimens, those on thiazides had the lowest observed median SBP $(134 \mathrm{mmHg})$, while those on ACE/ARB had the lowest observed median DBP $(82 \mathrm{mmHg})$. For non-BAA patients on 1-drug

Table 1. Sociodemographic and Baseline Characteristics

\begin{tabular}{|c|c|c|c|c|}
\hline Characteristic & $\begin{array}{l}\text { Total Population } \\
\mathrm{N}=10,875(\%)\end{array}$ & $\begin{array}{c}\text { Black/African American } \\
\text { Population } \\
\mathrm{N}=2,245(\%)\end{array}$ & $\begin{array}{c}\text { Non-BAA Population } \\
\mathrm{N}=8,630(\%)\end{array}$ & $P$ value \\
\hline Age $($ mean + SD) & $56.2+12.0$ & $52.4+11.4$ & $57.1+12.0$ & $<0.001^{*}$ \\
\hline Sex (female) & $6289(57.8)$ & $1262(56.2)$ & $5027(58.3)$ & $0.082 \dagger$ \\
\hline \# of PCP visits & & & & $0.040 \dagger$ \\
\hline 1 to 2 & $3825(36.9)$ & $752(35.1)$ & $3073(37.3)$ & \\
\hline 3 to 4 & $2853(27.5)$ & $579(27.0)$ & $2274(27.6)$ & \\
\hline 5 or more visits & $3695(35.6)$ & $812(37.9)$ & $2883(35.0)$ & \\
\hline Insurance status & & & & $0.001 \dagger$ \\
\hline Insured & $10226(94.0)$ & $2059(91.7)$ & $8167(94.6)$ & \\
\hline Uninsured & $585(5.4)$ & $172(7.7)$ & $413(4.8)$ & \\
\hline Other/unknown & $64(0.6)$ & $14(0.6)$ & $50(0.6)$ & \\
\hline Current Smoker & $1991(18.3)$ & $869(38.7)$ & $1122(13.0)$ & $0.001 \dagger$ \\
\hline BMI & & & & $<0.001 \dagger$ \\
\hline Less than 25 & $2915(27.7)$ & $436(20.2)$ & $2479(29.7)$ & \\
\hline 25 to 30 & $3554(33.8)$ & $596(27.6)$ & $2958(35.4)$ & \\
\hline$>30$ & $4038(38.4)$ & $1126(52.2)$ & $2912(34.9)$ & \\
\hline Language (English) & 7995 (73.4) & $2175(97.0)$ & $5800(67.3)$ & $<0.001 \dagger$ \\
\hline Clinical Site & & & & $<0.001 \dagger$ \\
\hline Health System 1 & $3362(30.9)$ & 391 (17.4) & $2971(34.4)$ & \\
\hline Health system 2 & $3710(34.1)$ & $886(39.5)$ & $2824(32.7)$ & \\
\hline Health System 3 & $3803(35.0)$ & $968(43.1)$ & $2835(32.9)$ & \\
\hline
\end{tabular}

Abbreviations: BAA, Black/African American; BMI, body mass index; PCP, primary care physician; SD, standard deviation. *T-Test.

†Pearson's Chi-Square Test. 
Table 2. Pharmacological Treatment Characteristics

\begin{tabular}{lccc}
\hline & $\begin{array}{c}\text { Total Population } \\
\mathrm{N}=10,875(\%)\end{array}$ & $\begin{array}{c}\text { Black/African American } \\
\text { Population } \\
\mathrm{N}=2,245(\%)\end{array}$ & $\begin{array}{c}\text { Non-BAA Population } \\
\mathrm{N}=8,630(\%)\end{array}$ \\
\hline $\begin{array}{l}\text { \# of drug classes } \\
1 \text { drug }\end{array}$ & & & $5904(68.4)$ \\
2 drugs & $7292(67.1)$ & $1388(61.8)$ & $2726(31.6)$ \\
1-Drug Regimens & $3583(32.9)$ & $857(38.2)$ & $2496(42.3)$ \\
ACE or ARB only & & $258(18.6)$ & $1633(27.7)$ \\
Thiazide only & $2754(37.8)$ & $573(41.3)$ & $1775(30.1)$ \\
CCB only & $2206(30.3)$ & $557(40.1)$ & $5.001^{*}$ \\
2-Drug Regimens & $2332(32.0)$ & & $539(19.8)$ \\
Thiazide + CCB & & $307(35.8)$ & $1347(49.4)$ \\
Thiazide + ACE/ARB & $846(23.6)$ & $380(44.3)$ & $840(30.8)$ \\
CCB + ACE/ARB & $1727(48.2)$ & $170(19.8)$ & $0.001^{*}$ \\
\hline
\end{tabular}

Abbreviations: BAA, Black/African American; CCB, Calcium Channel Blocker; ACE, Angiotensin-Converting Enzyme Inhibitor; ARB, Angiotensin Receptor Blocker.

*Pearson's Chi-Square Test.

regimens, those on ACE/ARB had the lowest observed median SBP $(133 \mathrm{mmHg})$, and those on CCB and ACE/ARB had the lowest observed median DBP $(79 \mathrm{mmHg})$. For BAA patients on 2 drug regimens, those on thiazide+ACE/ARB had the lowest observed median SBP $(135 \mathrm{mmHg})$ while those on $\mathrm{CCB}+\mathrm{ACE} / \mathrm{ARB}$ had the lowest observed median DBP $82 \mathrm{mmHg}$ ). Among non-BAA patients on 2 drugs, those on thiazide+ACE/ARB had the lowest observed median systolic BP $(132 \mathrm{mmHg})$, while those on $\mathrm{CCB}+\mathrm{ACE} / \mathrm{ARB}$ had the lowest observed median diastolic $\mathrm{BP}(78 \mathrm{mmHg})$.

\section{HTN Control by Race}

Overall, more of the BAA patients had uncontrolled HTN (BP $\geq 140 / 90 \mathrm{mmh}$ ) compared with the nonBAA patients (46.4\%; $95 \%$ CI, $0.44-0.48$ vs $39.0 \%$; 95\%CI, $0.38-0.40 ; P<.001)$. Of BAA patients on 1-drug regimens, $45.2 \%$ (95\% CI, 0.43-0.48) had uncontrolled HTN compared with 38.0\% (95\% CI, $0.37-0.39)$ of non-BAA $(P<.001)$. Of BAA patients on 2-drug regimens, $48.2 \%$ (95\% CI, 0.45-0.52) had uncontrolled HTN compared with 41.1\% (95\% CI, $0.39-0.43)$ of non-BAA $(P<.0001)$.

\section{Odds of Uncontrolled Blood Pressure among BAA Patients}

In our adjusted models, we found that BAA patients on 1-drug regimens including $\mathrm{CCB}$ and $\mathrm{ACE} / \mathrm{ARB}$ medications had higher odds of uncontrolled HTN compared with BAA patients on thiazides (adjusted odds ratio $[\mathrm{aOR}]=1.53 ; 95 \% \mathrm{CI}, 1.19-1.97$ and ${ }_{\mathrm{aOR}}=1.70 ; 95 \% \mathrm{CI}, 1.25-2.33$, respectively). Of BAA patients on 2-drug regimens, there were no significant differences between the 3 treatment combinations. Of note, BAA patients with more than 2 visits to their primary care providers during the study period had significantly lower odds of uncontrolled HTN than those with only 1 to 2 visits. (Table 3 )

\section{Discussion}

Our study revealed that prescribing practices for uncomplicated HTN were markedly different for BAA versus non-BAA populations in the health systems we examined, with a strong preference for thiazide diuretics and $\mathrm{CCB}$ as initial monotherapy and relatively less use of ACE/ARB in 1-drug or 2drug regimens for BAA patients. This indicates that primary care clinicians in these settings have, to a large extent, been practicing in accord with racebased HTN treatment guidelines that have been developed and disseminated in the last decade. Despite these findings, we also found evidence of continued disparities in HTN control for the BAA group relative to those in the non-BAA group, regardless of the treatment selected. Though BAA patients in this cohort who were prescribed thiazides did have better blood pressure control on average than those on monotherapy with other medications, we did not observe such benefits for individuals prescribed $\mathrm{CCB}$, and across all drug regimens for both BAA and non-BAA, there was far 
Figure 1a) Median and interquartile ranges of blood pressures for Black/African Americans and Non-Black/ African Americans on 1-drug regimens. Figure 1b) Median and interquartile ranges of blood pressures for Black/African Americans and Non-Black/African Americans on 2-drug regimens. Abbreviations: BP, Blood Pressure; CCB, Calcium Channel Blocker; ACE, Angiotensin-Converting Enzyme Inhibitor; ARB, Angiotensin Receptor Blocker.
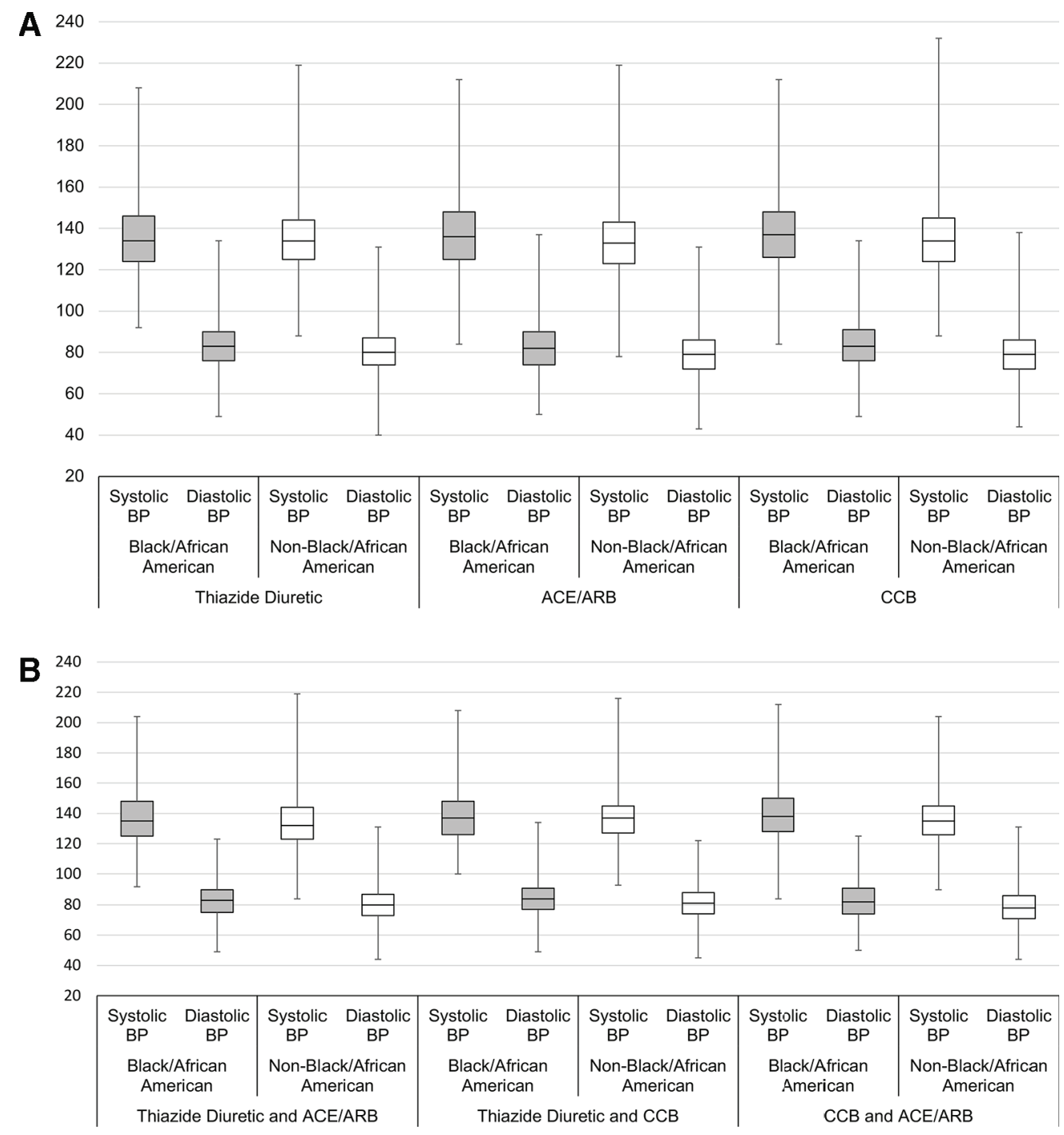

In the box plots, the boundary of the box indicates the 25 th and 75 th percentile. The solid black line indicates the median. The whiskers represent the minimum and maximal blood pressures.

more variation within groups than between groups, suggesting that response to medication prescribed is highly individual, regardless of race.

Our findings suggest that race-based hypertension prescribing guidelines have influenced clinical practice but likely with relatively limited benefit for BAA patients. Other factors may be more important than choice of drug based on skin colors, such as dose titration, the addition of second or third drugs, medication adherence, dietary and lifestyle interventions, such as advocating for smoking cessation, reducing alcohol consumption, and achieving weight loss. Our finding that more frequent clinical follow-up was associated with hypertension control among the BAA patients suggests that there may have been more time during more frequent visits to attend to these important clinical factors.

In addition, social and environmental factors that can contribute to hypertension disparities, such as lack of access to healthy food, unstable housing, social isolation, difficulties paying bills, may also be important risk factors that deserve greater 
Table 3. Odds of Uncontrolled Blood Pressure ( $\geq 140 / 90 \mathrm{mmHg})$ Among Black/African American Patients on Oneand Two-Drug anti-Hypertensive Regimens

\begin{tabular}{|c|c|c|c|c|}
\hline \multirow[b]{2}{*}{ Predictor } & \multicolumn{2}{|c|}{ One Drug Regimen $(\mathrm{n}=1327)$} & \multicolumn{2}{|c|}{ Two Drug Regimen $(\mathrm{n}=815)$} \\
\hline & $\mathrm{aOR}(95 \% \mathrm{CI})^{*}$ & $P$ value & $\mathrm{aOR}(95 \% \mathrm{CI})^{*}$ & $P$ value \\
\hline Age & $0.99(0.98-1.00)$ & 0.03 & $0.98(0.97-1.00)$ & 0.03 \\
\hline \multicolumn{5}{|l|}{ 1-Drug Regimen } \\
\hline Thiazide & 1.00 & Referent & & \\
\hline Calcium Channel Blocker & $1.53(1.19-1.97)$ & 0.001 & & \\
\hline ACE/ARB & $1.70(1.25-2.33)$ & 0.001 & & \\
\hline \multicolumn{5}{|l|}{ 2-Drug Regimen } \\
\hline Thiazide + CCB & & & 1.00 & Referent \\
\hline Thiazide + ACE/ARB & & & $0.81(0.59-1.12)$ & 0.20 \\
\hline $\mathrm{CCB}+\mathrm{ACE} / \mathrm{ARB}$ & & & $1.12(0.74-1.68)$ & 0.59 \\
\hline \multicolumn{5}{|l|}{ Sex } \\
\hline Female & 1.00 & Referent & 1.00 & Referent \\
\hline Male & $0.83(0.66-1.04)$ & 0.10 & $0.82(0.61-1.10)$ & 0.18 \\
\hline \multicolumn{5}{|l|}{ Insurance Status } \\
\hline Insured & 1.00 & Referent & 1.00 & Referent \\
\hline Uninsured & $1.13(0.75-1.73)$ & 0.55 & $0.91(0.52-1.60)$ & 0.75 \\
\hline Other/Unknown & $1.89(0.43-8.37)$ & 0.40 & $0.54(0.09-3.34)$ & 0.51 \\
\hline \multicolumn{5}{|l|}{ No. of PCP Visits } \\
\hline 1 to 2 & 1.00 & Referent & 1.00 & Referent \\
\hline 3 to 4 & $0.71(0.53-0.94)$ & 0.02 & $0.42(0.29-0.62)$ & $<0.0001$ \\
\hline 5 or more visits & $0.57(0.43-0.75)$ & $<0.0001$ & $0.48(0.34-0.67)$ & $<0.0001$ \\
\hline \multicolumn{5}{|l|}{ Clinical Site } \\
\hline Health System 1 & 1.00 & Referent & 1.00 & Referent \\
\hline Health System 2 & $2.04(1.56-2.66)$ & $<0.0001$ & $2.31(1.64-3.25)$ & $<0.0001$ \\
\hline Health System 3 & $1.59(1.15-2.20)$ & $<0.0001$ & $1.40(0.90-2.19)$ & 0.13 \\
\hline
\end{tabular}

Abbreviations: ACE, Angiotensin-Converting Enzyme Inhibitor; aOR, adjusted odds ratio; ARB, Angiotensin receptor blocker; BAA, Black/African American; CCB, Calcium Channel Blocker; CI, confidence interval; PCP, primary care physician.

*Adjusted for all variables included in the table.

attention. ${ }^{15}$ Finally, a factor that deserves specific focus is the role of implicit bias in medicine. It has long been known that race plays an implicit and often negative influence on clinician decision-making. ${ }^{27}$ Many studies have shown that BAA often receives poorer care and less referrals than nonBAA populations. ${ }^{27-30}$ At the least, race-specific medication guidelines provide a poor proxy for precision medicine, and at worst they may reinforce negative stereotypes suggesting that BAA patients are both genetically different from other races and that they should therefore be presented with a more limited set of treatment options. These guidelines may also distract clinicians from a more important type of precision medicine, including targeted interventions that address known social determinants of health and a clinician's own implicit biases that disproportionately and negatively impact BAA patients. ${ }^{31-34}$ Furthermore, use of these race-based HTN guidelines could have negative clinical consequences for BAA patients. For example, BAA has up to 4 times the prevalence of $\mathrm{CKD}$, and 1 potential reason could be delayed use of ACE/ARB medications in the early stages of CKD due to reliance on race-based clinical guidelines. ${ }^{35}$ Further study is needed to understand if preferentially avoiding ACE/ARB in BAA could contribute to well-documented disparities in long-term HTN outcomes. Finally, BAA patients do not make up a monolithic group; instead, individuals present a rich array of experiences and circumstance, and each person deserves to be treated as a unique individual. ${ }^{36,37}$ Instead of using race as a proxy for precision medicine, we should be searching for more biologically sound pharmacogenomic approaches that are precise and individualized. 
Our study, which relies on cross-sectional clinical data, had several limitations. First, our study used EHR reported race/ethnicity. EHR reported race often underestimates BAA race when compared with self-report. ${ }^{38}$ Next, we may not have excluded all individuals with comorbidities from our analyses, as the accuracy of such data in the EHR is dependent on clinician documentation. In addition, in our analyses, we did not have access to drug doses or changes in medication prescribing patterns that may have taken place over time. Furthermore, medication lists can be inaccurate due to poor documentation. Another important limitation is this study's inability to control how blood pressure was measured. There are potential differences in how individual health care providers and clinics within and across the health systems collect blood pressure readings. This inconsistency could be reflected in our data. Another consideration is medication adherence. EHR data cannot measure adherence to medication regimens accurately, and this study was not aiming to estimate adherence to medications. ${ }^{39}$ However, while these EHR-related limitations are real, they should be evenly spread across the entire cohort and equally distributed across BAA and the non-BAA subgroups. Finally, though the population studied was socioeconomically and racially heterogeneous, our study was conducted in the San Francisco Bay Area, and our findings may not be generalizable to other geographical areas in the United States. Future studies could address these limitations to provide more definitive conclusions to our research questions.

In conclusion, our study shows that while primary care providers often seem to follow racebased prescribing recommendations for HTN, disparities in HTN control for BAA patients relative to other populations persist. While racebased HTN guidelines are well-intentioned, now is the time for more research to understand better the clinical outcomes and possible unintended negative consequences of such guidelines for individuals they are intended to help.

The authors would like to acknowledge the clinical leaders and medical informatics teams at each health system without whose participation and assistance this research would not have been possible.

To see this article online, please go to: http://jabfm.org/content/ 35/1/26.full.

\section{References}

1. Caulfield T, Fullerton SM, Ali-Khan SE, et al. Race and ancestry in biomedical research: exploring the challenges. Genome Med 2009;1:8.

2. Roberts DE. Is race-based medicine good for us?: African American approaches to race, biomedicine, and equality. J Law Med Ethics 2008;36:537-45.

3. Smart A, Weiner K. Racialised prescribing: enacting race/ethnicity in clinical practice guidelines and in accounts of clinical practice. Sociol Health Illn 2018;40:843-58.

4. Cerdeña JP, Plaisime MV, Tsai J. From race-based to race-conscious medicine: how anti-racist uprisings call us to act. The Lancet 2020;396:1125-8.

5. Westby AO, Ricco J. Race-based treatment decisions perpetuate structural racism. Am Fam Physician 2020. Available at: https://www.aafp.org/ afp/2020/0801/p136.html. Accessed Nov 9, 2020.

6. Grubbs V. Precision in GFR Reporting. Clin J Am Soc Nephrol 2020;15:1201-2.:CJN.00690120.

7. Goodman CW, Brett AS. Race and Pharmacogenomics-Personalized Medicine or Misguided Practice? JAMA 2021;325:625.

8. Braun L, Fausto-Sterling A, Fullwiley D, et al. Racial categories in medical practice: how useful are they? PLoS Med 2007;4:e271.

9. Corbie-Smith G, Henderson G, Blumenthal C, Dorrance J, Estroff S. Conceptualizing race in research. J Nat Med Assoc 2008;100:1235-43.

10. Reddick B. Fallacies and dangers of practicing racebased medicine. Am Fam Physician 2021;104:122-3. Aug 1.

11. Grossman DC, Curry SJ, Owens DK, US Preventive Services Task Force, , et al. Screening for prostate cancer. JAMA 2018;319:1901-13.

12. Noor Chadha BL, Madelene KB. Rowland Toward the Abolition of Biological Race in Medicine. Institute OB; 2020. May 13, 2020.

13. Vyas DA, Eisenstein LG, Jones DS. Hidden in plain sight - reconsidering the use of race correction in clinical algorithms. N Engl J Med 2020;383:874-82.

14. Powe NR. Black kidney function matters: use or misuse of race? JAMA 2020;324:737-8.

15. Williams SK, Ravenell J, Seyedali S, Nayef H, Ogedegbe G. Hypertension treatment in blacks: discussion of the U.S. clinical practice guidelines. Prog Cardiovasc Dis 2016;59:282-8.

16. James PA, Oparil S, Carter BL, et al. 2014 evidencebased guideline for the management of high blood pressure in adults: report from the panel members appointed to the Eighth Joint National Committee (JNC 8). JAMA2014;311:507-20. Feb 5.

17. Whelton PK, Carey RM, Aronow WS, et al. 2017 ACC/AHA/AAPA/ABC/ACPM/AGS/APhA/ASH/ ASPC/NMA/PCNA Guideline for the Prevention, Detection, Evaluation, and Management of High 
Blood Pressure in Adults: A Report of the American College of Cardiology/American Heart Association Task Force on Clinical Pr. Hypertension 2018;71: e13-e115.

18. Williams B, Mancia G, Spiering W, ESC Scientific Document Group, et al. 2018 ESC/ESH Guidelines for the management of arterial hypertension. Eur Heart J 2018;39:3021-104.

19. Flack JM, Sica DA, Bakris G, et al. Management of high blood pressure in blacks. Hypertension 2010;56: 780-800.

20. Benjamin EJ, Muntner P, Alonso A, On behalf of the American Heart Association Council on Epidemiology and Prevention Statistics Committee and Stroke Statistics Subcommittee, , et al. Heart Disease and Stroke Statistics-2019 Update: A Report From the American Heart Association. Circulation 2019;139.

21. Musemwa N, Gadegbeku CA. Hypertension in African Americans. Curr Cardiol Rep 2017;19.

22. Laragh JH, Sealey JE. Relevance of the plasma renin hormonal control system that regulates blood pressure and sodium balance for correctly treating hypertension and for evaluating ALLHAT., Am J Hypertens 2003;16:407-15.

23. Wright JT, Bakris G, Greene T, African American Study of Kidney Disease and Hypertension Study Group, et al Effect of blood pressure lowering and antihypertensive drug class on progression of hypertensive kidney disease, results from the AASK Trial. JAMA 2002;288:2421-31.

24. Madhusoodanan J. Is a racially-biased algorithm delaying health care for one million Black people? Nature 2020;588:546-7.

25. Selby K, Michel M, Gildengorin G, et al. Disparities in hypertension control across and within three health systems participating in a data-sharing collaborative. J Am Board Fam Med 2018;31:897-904.

26. Benchimol EI, Smeeth L, Guttmann A, RECORD Working Committee, , et al. The REporting of studies Conducted using Observational Routinelycollected health Data (RECORD) statement. PLOS Med 2015;12:e1001885.

27. FitzGerald C, Hurst S. Implicit bias in healthcare professionals: a systematic review. BMC Med Ethics. 2017;18:19 2017/03/01.
28. Schulman KA, Berlin JA, Harless $W$, et al. The effect of race and sex on physicians' recommendations for cardiac catheterization. $\mathrm{N}$ Engl J Med 1999;340:618-26.

29. Ravenell J, Ogedegbe G. Unconscious bias and realworld hypertension outcomes: advancing disparities research. J Gen Intern Med 2014;29:973-5. Jul.

30. Nelson A. Unequal treatment: confronting racial and ethnic disparities in health care. J Natl Med Assoc 2002;94:666-8.

31. Adler NE, Glymour MM, Fielding J. Addressing social determinants of health and health inequalities. JAMA 2016;316:1641-2.

32. Chetty R, Stepner M, Abraham S, et al. The association between income and life expectancy in the United States, 2001-2014. JAMA 2016;315: 1750-66.

33. De Mendoza VB, Huang Y, Crusto CA, Sun YV, Taylor JY. Perceived racial discrimination and DNA methylation among African American women in the InterGEN Study. Biological Research For Nursing 2018;20:145-52.

34. Gruenewald TL, Karlamangla AS, Hu P, et al. History of socioeconomic disadvantage and allostatic load in later life. Soc Sci Med 2012;74:75-83.

35. Chronic Kidney Disease Surveillance SystemUnited States. website. Center for Disease Control and Prevention. Available at: http://www.cdc.gov/ ckd. Accessed March 18, 2021.

36. Celious A, Oyserman D. Race from the inside: an emerging heterogeneous race model. J Social Isssues 2001;57:149-65.

37. McKinley LA, Smith JR, Marshall KP. The Myth of the Monolithic Minority: The Renewed Call for Effective Segmentation of African American Consumers. Springer International Publishing; 2015: 92-9.

38. Klinger EV, Carlini SV, Gonzalez I, et al. Accuracy of race, ethnicity, and language preference in an electronic health record. J GEN INTERN MED 2015;30:719-23.

39. Galozy A, Nowaczyk S, Sant'Anna A, Ohlsson M, Lingman M. Pitfalls of medication adherence approximation through EHR and pharmacy records: definitions, data and computation. International Journal of Medical Informatics 2020;136:104092. 